\title{
Numerical analysis of a cell dwarfism model
}

\author{
L.M. Abia ${ }^{b}$, O. Angulo ${ }^{a, *}$, J.C. López-Marcos ${ }^{b}$, M.A. López-Marcos ${ }^{b}$ \\ a Departamento de Matemática Aplicada. ETSIT. Universidad de Valladolid. Pso. \\ Belén 5, 47011 Valladolid. SPAIN. Phone: +34983 423000(Ext: 5835). FAX: +34 983 \\ 423661. \\ ${ }^{b}$ Departamento de Matemática Aplicada. Facultad de Ciencias. Universidad de \\ Valladolid. Pso. Belén 7, 47011 Valladolid. SPAIN.
}

\begin{abstract}
In this work, we study numerically a model which describes cell dwarfism. It consists in a pure initial value problem for a first order partial differential equation, that can be applied to the description of the evolution of diseases as thalassemia. We design two numerical methods that prevent the use of the characteristic curve $x=0$, and derive their optimal rates of convergence. $\mathrm{Nu}-$ merical experiments are also reported in order to demonstrate the predicted accuracy of the schemes. Finally, a comparison study on their efficiency is presented.
\end{abstract}

Keywords: Size-structured population; cell population models; dwarfism; numerical methods; convergence analysis; efficiency

2000 MSC: 92D25, 92D40, 65M25, 65M12, 35B40

\section{Introduction}

We analyze, from a numerical point of view, a cell population balance model (CPBM) in which cells are distinguished by their individual size. CPBM were introduced in the early 1960s within the framework of particle dynamics in chemical and cellular contexts $[17,6,16]$. From a formal point of view, CPBM can be defined as the balance equation that accounts for the various processes that change the number of cells in a population. In general, it takes the form of a first-order integro-partial differential equation, along with boundary and initial condition. From a theoretical point of view, mathematical treatment of linear CPBMs has been developed since the early

\footnotetext{
${ }^{*}$ Corresponding author

Email addresses: abia@mac.uva.es (L.M. Abia), oscar@mat.uva.es (O. Angulo), lopezmar@mac.uva.es (J.C. López-Marcos), malm@mac.uva.es (M.A. López-Marcos)
} 
1980s $[7,12,10]$, where the study of the well-posedness, the convergence towards an asymptotically stable-size distribution and the stability analysis were made. In the case of a nonlinear model, the theoretical properties of existence and uniqueness of solutions have been adressed in [12].

The CPBM that we consider in this paper is based upon the model developed by Diekmann et al. [7], where cell-size is used to distinguish individuals in the population. We use the version presented in [14],

$$
\begin{aligned}
& u_{t}(x, t)+(x u(x, t))_{x}=(\nu(x)-\mu(x)-b(x)) u(x, t)+4 b(2 x) u(2 x, t) \\
& 0<x<1, t>0 \\
& u(x, 0)=\varphi(x), \quad 0<x \leq 1
\end{aligned}
$$

where the population of cells is described by a density function $u(x, t), t$ represents time and $x$ measure the cell-size. Functions $\mu, b$ and $\nu$ explain different processes which take place within the population. In this model, cells grow exponentially, $x^{\prime}(t)=x(t)$, as in a petri dish experiment, and die with death rate $\mu(x)$ depending on cellular size. With respect to the division process, we have considered a division in which the mother cell splits into two equal cells [7]. Note that the exponential growth introduces the unavailability of a boundary condition at size $x=0$, thus cell renewal is introduced through the division $b(x)$ and immigration $\nu(x)$ rates. If we deal with a closed system (petri dish), it is usual to consider $\nu(x)=0$, however other biologically significant systems are not closed, for example the blood production system which needs to replaced the red blood cells daily in order to regulate the blood cells count (stem cell regulation). We want to point out that a proper combination of growth, division and mortality rates would introduce a natural maximum cell size [2], otherwise we could fix it as one (normalized) and we would consider that larger cells may only grow and die. As in [14], we assume $\mu$ and $b$ are both positive, uniformly continuous functions on $(0,1)$, with support in the interval $[0,1]$ and $\nu(x)$ is a nonnegative, uniformly continuous function bounded above in the interval $[0,1]$. We also assume that the environment is unlimited and all possible nonlinear mechanisms are ignored. Function $\varphi$ is the initial state of the population density.

The usual CPBM, as developed in [7], assumes that a cell does not divide until it reaches a minimal cell size $a>0$, which generates a minimal cellular size $a / 2$. However, model (1.1)-(1.2) allows a cell of any size in the interval $(0,1]$ to divide. Therefore, the minimal cellular size is $a=0$. Although the idea of a cell with zero size is biologically unrealistic, we use it as the limiting value to describe an abnormality in the cellular division process: the production of unfunctional "dwarf" cells. These kind of cells are observed in a group of inherit blood disorders that affect the body's ability 
to produce hemoglobin and red blood cells: thalassemia. These hereditary blood disorders (anemias) are one of the most common human genetic abnormalities known and they are prevalent in tropical and subtropical world regions where malaria is still epidemic. Major $\alpha$-thalassemia disorder ( $h y$ drops fetalis) has a high lethality rate and it has become an important public health problem due to population migrations. Besides, carriers of (minor) $\alpha$-thalassaemia are found at high frequencies and they are usually asymptomatics. The disorders are caused by the absent or decreased production of the $\alpha$ chain of hemoglobin. In healthy persons, the synthesis of $\alpha$ and $\beta$-globin chains is finely balanced during terminal erythroid differentiation, giving rise to red blood cells of consistent size (reflected in the mean corpuscular volume $(\mathrm{MCV})$ ) and hemoglobin content (mean corpuscular hemoglobin $(\mathrm{MCH})$ ). Thus, minor forms of thalassemia are associated with smaller red blood cells than normal, a condition known as microcytosis which are only distinguishable through MCV. Finally, these diseases also can be associated with other blood disorders as the myelodysplastic syndrome $[19,9,11]$.

Some theoretical properties of the model (1.1)-(1.2) were developed in [14]. In that work, the author addressed the existence and uniqueness of generalized solutions and their stability and unstability. On the one hand, he established the conditions on the data functions bounds to obtain a strongly stable solution, that biologically shows the extinction of the population. On the other hand, he proposed the data functions properties that leads to the topological transitivity of the different cellular generations. It includes the erratic behaviour customarily associated with chaos. Such an issue has been subsequently refined in $[8,18]$ and references therein.

These theoretical properties can be studied without a solution expression. However, the knowledge of their qualitative or quantitative behaviour in a more tangible way is sometimes necessary. Therefore, numerical methods provide a valuable tool to obtain such an information. In the case of general structured population models, many numerical methods have been proposed to solve them (see $[1,13]$ and references therein). With respect to the study of CPBMs, different techniques have been used for both symmetric and asymmetric division rates (see $[2,4,5,3]$ and the references therein). However, all of them are proposed for the solution of models with a minimal cell division size, and it is very important to design numerical schemes specially adapted to the features of this particular CPBM. For this model, there is an expression of the generalized solution but this formula does not possess an easy computational form, even in simple situations [14].

In this work, we present and analyze two first-order procedures: a natural grid method and an upwind scheme which are specially adapted to obtain the solution to the problem (1.1)-(1.2). 
In Section 2 we describe the proposed numerical methods. In Section 3 we analyze their convergence to the exact solution and, in Section 4, we carry out a representative numerical simulation, including a comparison of the efficiency of the methods.

\section{Numerical Methods}

We will introduce two numerical methods of first order adapted to different peculiarities of this particular model. On one hand, note that there is a characteristic curve at $x=0$. Therefore, we avoid to use this "unknown" information into our numerical schemes. On the other hand, the solution of the problem is only first-order continuously differentiable, therefore we elude higher order methods. The first proposal is based on the integration along the characteristic curves, the other one consists on a finite difference method connected to an upwind technique.

\subsection{Natural grid method (NGM)}

This numerical integration is based on the discretization of the solution along the characteristic curves. Therefore, we define $\mu^{*}(x)=1+\mu(x)+$ $b(x)-\nu(x)$ and rewrite $(1.1)$ as

$$
u_{t}(x, t)+x u_{x}(x, t)=-\mu^{*}(x) u(x, t)+4 b(2 x) u(2 x, t),
$$

$0<x<1, t>0$. We denote by $x\left(t ; t^{*}, x_{*}\right)=x_{*} \exp \left(t-t^{*}\right)$, the characteristic curve of the equation (2.1) (and (1.1)) which takes the value $x_{*} \neq 0$ at time $t^{*}$, and define $w\left(t ; t^{*}, x_{*}\right)=u\left(x\left(t ; t^{*}, x_{*}\right), t\right), t \geq t^{*}$. Thus

$$
\left\{\begin{aligned}
\frac{d}{d t} w\left(t ; t^{*}, x_{*}\right)= & -\mu^{*}\left(x\left(t ; t^{*}, x_{*}\right)\right) w\left(t ; t^{*}, x_{*}\right) \\
& +4 b\left(2 x\left(t ; t^{*}, x_{*}\right)\right) u\left(2 x\left(t ; t^{*}, x_{*}\right), t\right), \quad t>t^{*}, \\
w\left(t^{*} ; t^{*}, x_{*}\right)= & u\left(x_{*}, t^{*}\right),
\end{aligned}\right.
$$

and the solution to (2.2) can be written as (see [4])

$$
\begin{aligned}
& u\left(x\left(t ; t^{*}, x_{*}\right), t\right)=u\left(x_{*}, t^{*}\right) \exp \left\{-\int_{t^{*}}^{t} \mu^{*}\left(x\left(\tau ; t^{*}, x_{*}\right)\right) d \tau\right\} \\
& +4 \int_{t^{*}}^{t} \exp \left\{-\int_{\tau}^{t} \mu^{*}\left(x\left(s ; t^{*}, x_{*}\right)\right) d s\right\} b\left(2 x\left(\tau ; t^{*}, x_{*}\right)\right) u\left(2 x\left(\tau ; t^{*}, x_{*}\right), \tau\right) d \tau, t \geq t^{*} .
\end{aligned}
$$

We propose a numerical method based on a discretization of the integral representation (2.3) of the solution on a suitable mesh over the time and size variables. 
We consider the numerical integration of model (1.1)-(1.2) along a finite time interval $[0, T]$. Thus, given a positive integer $N$, we define the time step $k=T / N$, and introduce the discrete time levels as $t^{n}=n k, 0 \leq n \leq N$.

Natural grid methods employ a nonuniform grid, called natural grid, which was introduced and studied first in [15]. On one hand, it is invariant due to the autonomous character of the ODE that rules the characteristic curves, thus we compute this fixed mesh before the time procedure starts. On the other hand, there exist suitable constants to bound the distance to the border of the size-interval (see [1]). In this particular case, the explicit form of the characteristics and the autonomous feature of the problem allow us to generate the grid easier.

We define the following spatial grid: $X_{j}=\exp (-(J-j) k), 1 \leq j \leq J$, where the integer $J$ is chosen in order to satisfy the condition $X_{1} \leq K_{1} k(J>$ $1-\frac{1}{k} \log \left(K_{1} k\right)$, for $k$ small enough), $K_{1}$ is a suitable constant which does not depend on $k$ (we refer to [1] for further details on a numerical generation of the natural grid). Note that the points $\left(X_{j}, t^{n}\right)$ and $\left(X_{j+1}, t^{n+1}\right), 1 \leq j \leq J-1$, $0 \leq n \leq N-1$, belong to the same characteristic curve, where the subscript $j$ refers to the grid point $X_{j}$, and the superscript $n$ to the time level $t^{n}$.

Thus, we denote $u_{j}^{n}=u\left(X_{j}, t^{n}\right)$, and let $U_{j}^{n}$ be a numerical approximation to $u_{j}^{n}, 1 \leq j \leq J, 0 \leq n \leq N$. We propose a one-step method in order to obtain it: starting from an approximation to the initial data (1.2) of the problem (for instance, the grid restriction of $\varphi$ ), the numerical solution at a new time level is described in terms of the previous one. Such a general step is obtained by means of the following first-order discretization of (2.3). For $0 \leq n \leq N-1$,

$$
\begin{aligned}
& U_{j+1}^{n+1}=\left(U_{j}^{n}+4 k b\left(2 X_{j}\right) \bar{U}_{2 \cdot j}^{n}\right) \exp \left(-k \mu_{j}^{*}\right), \quad 1 \leq j \leq J-1, \\
& U_{1}^{n+1}=\left(U_{1}^{n}+4 k b\left(2 X_{1}^{*}\right) \bar{U}_{2 \cdot *}^{n}\right) \exp \left(-k \mu^{*}\left(X_{1}^{*}\right)\right),
\end{aligned}
$$

where $\mu_{j}^{*}=\mu^{*}\left(X_{j}\right), 1 \leq j \leq J$. The points $2 X_{j}, 1 \leq j \leq J-1$, do not belong to the natural grid, then the aproximation to $b\left(2 X_{j}\right) u\left(2 X_{j}, t^{n}\right)$ is given by: $b\left(2 X_{j}\right) \bar{U}_{2 \cdot j}^{n}=b\left(2 X_{j}\right) U_{M-1}^{n}$, if there exists $M$ such that $X_{M-1} \leq$ $2 X_{j}<X_{M} \leq 1$. Otherwise is zero. Notice that the calculus of $U_{1}^{n+1}$ is different because we do not use the value of the solution at the characteristic curve $x=0$. Therefore, we employ an extra grid node $X_{1}^{*}=X_{1} \exp (-k)$. We take $U_{1}^{n}$ as the approximation to $u\left(X_{1}^{*}, t^{n}\right)$, and, $\bar{U}_{2 \cdot *}^{n}=U_{M-1}^{n}$, where $M$ is such that $X_{M-1} \leq 2 X_{1}^{*} \leq X_{M} \leq 1$.

\subsection{Upwind method (UM)}

The following scheme is a finite difference method based on an upwind technique. Again, we consider the numerical integration of model (1.1)-(1.2) along a finite time interval $[0, T]$. 
First, we introduce a uniform grid on the size interval. Let $J$ be a positive integer, we consider the grid points $X_{j}=j h, 1 \leq j \leq 2 J$, where $h=\frac{1}{2 J}$ is the mesh size. Now, we fix a constant $r \in \mathbb{R}$, and $k=r h$, thus $N=[T / k]$, and introduce the discrete time levels as $t^{n}=n k, 0 \leq n \leq N$. Once more, the subscript $j$ refers to the grid point $X_{j}$, and the superscript $n$ to the time level $t^{n}$.

Again, we denote $u_{j}^{n}=u\left(X_{j}, t^{n}\right)$, and let $U_{j}^{n}$ be a numerical approximation to $u_{j}^{n}, 1 \leq j \leq 2 J, 0 \leq n \leq N$. As in the previous procedure, we consider an approximation to the initial data (1.2) (again, the grid restriction of $\varphi$ is valid). Now, we propose the following one step method that is obtained by discretizating the derivatives in (1.1) in the following way: for $0 \leq n \leq N-1$,

$$
\frac{U_{j}^{n+1}-U_{j}^{n}}{k}+X_{j} \frac{U_{j}^{n}-U_{j-1}^{n}}{h}=-\mu_{j}^{*} U_{j}^{n}+4 b_{2 j} U_{2 j}^{n}, \quad j=2, \ldots, 2 J
$$

where $\mu_{j}^{*}=\mu^{*}\left(X_{j}\right), b_{j}=b\left(X_{j}\right), 1 \leq j \leq 2 J$. As in the previous scheme, we do not use the value at $x=0$, therefore we compute an approximation to $u\left(X_{1}, t^{n+1}\right)$ by means of the discretization of $(2.3)$, in which the integral terms inside the exponential are approximated using the rectangule rule based on $t=t^{n+1}$,

$$
U_{1}^{n+1}=U_{1}^{n} \exp \left\{-k \mu_{1}^{*}\right\}+4 k b_{2} U_{2}^{n+1} .
$$

Remark 1. We want to highlight that both schemes preserve the non negativity property of the biological model. This feature is not conserved by other numerical methods adapted to the problem as, for example, the one that discretize with implicit upwind procedures.

Remark 2. The fact that the support of $b$ belongs to $[0,1]$ makes formulae in (2.4) and (2.6) to be implemented in a different way when the term $b(2 x) u(2 x, t)$ vanishes $(2 x>1)$.

\section{Convergence Analysis}

In this section, we carry out the convergence analysis of the schemes. It is based on their consistency and stability properties.

\subsection{Natural grid method}

If $u$ is the solution to problem (1.1)-(1.2), we define

$$
\mathbf{u}^{n}=\left(u_{1}^{n}, \ldots, u_{J}^{n}\right), u_{j}^{n}=u\left(X_{j}, t^{n}\right), 1 \leq j \leq J, 0 \leq n \leq N .
$$

We can write the numerical scheme as $\mathbf{U}^{n+1}=\mathcal{A}(k) \mathbf{U}^{n}, 0 \leq n \leq N-1$, where $\mathcal{A}(k)=\left(a_{i j}\right)_{i, j=1}^{J}$ is a sparse matrix: at each row, there are at most 
two nonzero entries. That is, for $1 \leq j \leq J-1, a_{j+1, j}=\exp \left(-k \mu_{j}^{*}\right)$, and, when there exists $M$ such that $X_{M-1} \leq 2 X_{j}<X_{M} \leq 1$, then $a_{j+1, M-1}=$ $4 k b\left(2 X_{j}\right) \exp \left(-k \mu_{j}^{*}\right)$. In the case of the first row, which has a different formula, $a_{11}=\exp \left(-k \mu^{*}\left(X_{1}^{*}\right)\right)$, and $a_{1, M-1}=4 k b\left(2 X_{1}^{*}\right) \exp \left(-k \mu^{*}\left(X_{1}^{*}\right)\right)$, where $M$ is such that $X_{M-1} \leq 2 X_{1}^{*}<X_{M} \leq 1$. Of course, $\mathbf{U}^{0}$ is the vector with the initial approximation to $\varphi$ in (1.2).

Thus we define, on the one hand, the local discretization error, $\boldsymbol{\tau}^{n+1}=$ $\left(\tau_{1}^{n+1}, \ldots, \tau_{J}^{n+1}\right), 0 \leq n \leq N-1$ as given by

$$
\boldsymbol{\tau}^{n+1}=\frac{1}{k}\left(\mathbf{u}^{n+1}-\mathcal{A}(k) \mathbf{u}^{n}\right), 0 \leq n \leq N-1 .
$$

On the other hand, the global discretization error as $\mathbf{E}^{n}=\left(E_{1}^{n}, \ldots, E_{J}^{n}\right)$, $E_{j}^{n}=u_{j}^{n}-U_{j}^{n}, 1 \leq j \leq J, 0 \leq n \leq N$. Thus,

$$
\mathbf{E}^{n+1}=\mathcal{A}(k) \mathbf{E}^{n}+k \boldsymbol{\tau}^{n+1}, \quad 0 \leq n \leq N-1 .
$$

The consistency analysis and the boundness of $\|\mathcal{A}(k)\|_{\infty}$ will give us the convergence. For a vector $\mathbf{v}=\left(v_{1}, \ldots, v_{J}\right)$, we denote by $\|\mathbf{v}\|_{\infty}$ its maximum norm. Henceforth, $C$ will denote a positive constant which is independent of $k, n(0 \leq n \leq N)$ and $j(1 \leq j \leq J)$; $C$ possibly has different values in different places.

Lemma 1 (Stability). Let $\mu^{*}$ have bounded and continuous first derivatives on $(0,1]$ and $b$ bounded on $(0,1]$. Then

$$
\|\mathcal{A}(k)\|_{\infty} \leq 1+C k
$$

for $k$ enough small.

Proof: $\|\mathcal{A}\|_{\infty}$ is defined as

$$
\|\mathcal{A}(k)\|_{\infty}=\max _{1 \leq i \leq J}\left\{\sum_{j=1}^{J}\left|a_{i j}\right|\right\} .
$$

Therefore,

$$
\begin{aligned}
\|\mathcal{A}(k)\|_{\infty} \leq & \max \left\{e^{-k \mu^{*}\left(X_{1}^{*}\right)}\left(1+4 k b\left(2 X_{1}^{*}\right)\right),\right. \\
& \left.\max _{1 \leq i \leq J^{*}-1}\left\{e^{-k \mu_{i}^{*}}\left(1+4 k b\left(2 X_{i}\right)\right)\right\}, \max _{J^{*} \leq i \leq J-1}\left\{e^{-k \mu_{i}^{*}}\right\}\right\},
\end{aligned}
$$


where $J^{*}$ is the minimum index that satisfies $2 X_{J^{*}} \geq 1$. Function $\mu^{*}$ is continuously differentiable and $b$ is bounded, so

$$
\left.e^{-k \mu_{i}^{*}} \leq 1+C k \quad \text { and } \quad 4 k b\left(2 X_{i}\right)\right) \leq C k, \quad 1 \leq i \leq J
$$

and we conclude the estimate (3.2). In fact, if $\nu(x)=0$, we could bound $e^{-k \mu_{i}^{*}} \leq 1,1 \leq i \leq J$.

Lemma 2 (Consistency). Let $\mu^{*}, b$ have bounded and continuous first derivatives on $(0,1]$, and $u$ have Lipschitz continuous first derivatives on $(0,1] \times$ $[0, T]$. Then, as $k \rightarrow 0$, the following estimates hold

$$
\left\|\boldsymbol{\tau}^{n+1}\right\|_{\infty}=O(k), \quad 0 \leq n \leq N-1 .
$$

Proof: From equations (2.4)-(2.5), we can write the components of the local discretization error as

$$
\begin{aligned}
\tau_{1}^{n+1} & =\frac{1}{k}\left(u_{1}^{n+1}-\left(u_{1}^{n}+4 k b\left(2 X_{1}^{*}\right) \bar{u}_{2 \cdot *}^{n}\right) e^{-k \mu^{*}\left(X_{1}^{*}\right)}\right), \\
\tau_{j+1}^{n+1} & =\frac{1}{k}\left(u_{j+1}^{n+1}-\left(u_{j}^{n}+4 k b\left(2 X_{j}\right) \bar{u}_{2 \cdot j}^{n}\right) e^{-k \mu_{j}^{*}}\right), 1 \leq j \leq J-1 .
\end{aligned}
$$

If we use equation (2.3) in (3.4), we obtain

$$
\begin{aligned}
& \tau_{1}^{n+1}=\frac{1}{k}\left(u\left(X_{1} e^{-k}, t^{n}\right) \exp \left\{-\int_{t^{n}}^{t^{n+1}} \mu^{*}\left(x\left(\tau ; t^{n}, X_{1} e^{-k}\right)\right) d \tau\right\}\right. \\
& +4 \int_{t^{n}}^{t^{n+1}} \exp \left\{-\int_{\tau}^{t^{n+1}} \mu^{*}\left(x\left(s ; t^{n}, X_{1} e^{-k}\right)\right) d s\right\} b\left(2 x\left(\tau ; t^{n}, X_{1} e^{-k}\right)\right) u\left(2 x\left(\tau ; t^{n}, X_{1} e^{-k}\right), \tau\right) d \tau \\
& \left.-\left(u_{1}^{n}+4 k b\left(2 X_{1} e^{-k}\right) \bar{u}_{2 \cdot *}^{n}\right) e^{-k \mu^{*}\left(X_{1}\right)}\right) .
\end{aligned}
$$

Due to the regularity hypothesis on $\mu^{*}, b$ and $u$, the convergence properties of the rectangular quadrature rule, the mean value theorem and that $X_{1} \leq C k$,

$$
\begin{aligned}
& \left|\tau_{1}^{n+1}\right| \leq \frac{1}{k}\left|u\left(X_{1} e^{-k}, t^{n}\right)-u\left(X_{1}, t^{n}\right)\right| e^{-k \mu^{*}\left(X_{1}^{*}\right)} \\
& \quad+\frac{1}{k} u\left(X_{1} e^{-k}, t^{n}\right) \mid \exp \left\{-\int_{t^{n}}^{t^{n+1}} \mu^{*}\left(x\left(\tau ; t^{n}, X_{1} e^{-k}\right)\right) d \tau\right\}-e^{-k \mu^{*}\left(X_{1}^{*}\right) \mid} \\
& \quad+\frac{4}{k} \mid \int_{t^{n}}^{t^{n+1}} \exp \left\{-\int_{\tau}^{t^{n+1}} \mu^{*}\left(x\left(s ; t^{n}, X_{1} e^{-k}\right)\right) d s\right\} b\left(2 x\left(\tau ; t^{n}, X_{1} e^{-k}\right)\right) u\left(2 x\left(\tau ; t^{n}, X_{1} e^{-k}\right), \tau\right) d \tau
\end{aligned}
$$




$$
\begin{aligned}
& -k \exp \left\{-\int_{t^{n}}^{t^{n+1}} \mu^{*}\left(x\left(s ; t^{n}, X_{1} e^{-k}\right)\right) d s\right\} b\left(2 X_{1} e^{-k}\right) u\left(2 X_{1} e^{-k}, t^{n}\right) \\
& +4\left|\exp \left\{-\int_{t^{n}}^{t^{n+1}} \mu^{*}\left(x\left(s ; t^{n}, X_{1} e^{-k}\right)\right) d s\right\}-e^{-k \mu^{*}\left(X_{1}^{*}\right) \mid}\right| b\left(2 X_{1} e^{-k}\right) u\left(2 X_{1} e^{-k}, t^{n}\right) \\
& +4 e^{-k \mu^{*}\left(X_{1}^{*}\right)}\left|b\left(2 X_{1} e^{-k}\right) u\left(2 X_{1} e^{-k}, t^{n}\right)-b\left(2 X_{1}\right) \bar{u}_{2 \cdot *}^{n}\right| \\
& \leq \frac{C}{k} X_{1}\left(1-e^{-k}\right)(1+C k)+C k+C(1+C k) X_{1}\left(1-e^{-k}\right) \\
& \leq C k \text {. }
\end{aligned}
$$

Now, we use equation (2.3) in (3.5) to obtain

$$
\begin{aligned}
& \tau_{j+1}^{n+1}=\frac{1}{k}\left(u\left(X_{j}, t^{n}\right) \exp \left\{-\int_{t^{n}}^{t^{n+1}} \mu^{*}\left(x\left(\tau ; t^{n}, X_{j}\right)\right) d \tau\right\}-u_{j}^{n} e^{-k \mu_{j}^{*}}\right. \\
& +4 \int_{t^{n}}^{t^{n+1}} \exp \left\{-\int_{\tau}^{t^{n+1}} \mu^{*}\left(x\left(s ; t^{n}, X_{j}\right)\right) d s\right\} b\left(2 x\left(\tau ; t^{n}, X_{j}\right)\right) u\left(2 x\left(\tau ; t^{n}, X_{j}\right), \tau\right) d \tau \\
& \left.-4 k b\left(2 X_{j}\right) \bar{u}_{2 \cdot j}^{n} e^{-k \mu_{j}^{*}}\right), 1 \leq j \leq J-1 .
\end{aligned}
$$

And, with the use of the same previous arguments and taking into account that $X_{j+1}-X_{j} \leq C k, 1 \leq j \leq J-1$, we arrive at

$$
\begin{aligned}
& \left|\tau_{j+1}^{n+1}\right| \leq \frac{1}{k} u\left(X_{j}, t^{n}\right)\left|\exp \left\{-\int_{t^{n}}^{t^{n+1}} \mu^{*}\left(x\left(\tau ; t^{n}, X_{j}\right)\right) d \tau\right\}-e^{-k \mu_{j}^{*}}\right| \\
& +\frac{4}{k} \mid \int_{t^{n}}^{t^{n+1}} \exp \left\{-\int_{\tau}^{t^{n+1}} \mu^{*}\left(x\left(s ; t^{n}, X_{j}\right)\right) d s\right\} b\left(2 x\left(\tau ; t^{n}, X_{j}\right)\right) u\left(2 x\left(\tau ; t^{n}, X_{j}\right), \tau\right) d \tau \\
& \quad-k \exp \left\{-\int_{t^{n}}^{t^{n+1}} \mu^{*}\left(x\left(s ; t^{n}, X_{j}\right)\right) d s\right\} b\left(2 X_{j}\right) u\left(2 X_{j}, t^{n}\right) \mid \\
& +4\left|\exp \left\{-\int_{t^{n}}^{t^{n+1}} \mu^{*}\left(x\left(s ; t^{n}, X_{j}\right)\right) d s\right\}-e^{-k \mu_{j}^{*}}\right| b\left(2 X_{j}\right) u\left(2 X_{j}, t^{n}\right) \\
& \quad+4 e^{-k \mu_{j}^{*}} b\left(2 X_{j}\right)\left|u\left(2 X_{j}, t^{n}\right)-\bar{u}_{2 \cdot j}^{n}\right| \\
& \leq C k .
\end{aligned}
$$

Thus, inequalities (3.6)-(3.7) allow us to arrive to the estimation (3.3)

In the following result, we prove the convergence of the numerical method. 
Theorem 3 (Convergence). Under the hypotheses of Lemma 2, if $\left\|\mathbf{E}^{0}\right\|_{\infty}=$ $O(k)$, as $k \rightarrow 0$, then

$$
\left\|\mathbf{E}^{n}\right\|_{\infty}=O(k), \quad 0 \leq n \leq N,
$$

as $k \rightarrow 0$.

Proof: From the definition of the global discretization error, we have

$$
\mathbf{E}^{n}=\mathcal{A}(k) \mathbf{E}^{n-1}+k \boldsymbol{\tau}^{n}=\mathcal{A}(k)^{n} \mathbf{E}^{0}+k \sum_{l=0}^{n-1} \mathcal{A}(k)^{l} \boldsymbol{\tau}^{n-l}, 1 \leq n \leq N .
$$

Thus

$$
\left\|\mathbf{E}^{n}\right\|_{\infty} \leq\left(\|\mathcal{A}(k)\|_{\infty}\right)^{n}\left\|\mathbf{E}^{0}\right\|_{\infty}+k \sum_{l=0}^{n-1}\left(\|\mathcal{A}(k)\|_{\infty}\right)^{l}\left\|\boldsymbol{\tau}^{n-l}\right\|_{\infty}, 1 \leq n \leq N .
$$

Therefore, the use of (3.2) and (3.3), produces (3.8).

\subsection{Upwind method}

Now, we define, again,

$$
\mathbf{u}^{n}=\left(u_{1}^{n}, \ldots, u_{2 J}^{n}\right), u_{j}^{n}=u\left(X_{j}, t^{n}\right), 1 \leq j \leq 2 J, 0 \leq n \leq N,
$$

where $u$ is the solution to problem (1.1)-(1.2). We write the numerical scheme as $\mathbf{U}^{n+1}=\mathcal{A}(k, h) \mathbf{U}^{n}, 0 \leq n \leq N-1$, where $\mathcal{A}(k, h)=\left(a_{i j}\right)_{i, j=1}^{2 J}$ is again a sparse matrix, in this case with at most three non zero entries at each row. It is defined as, for $2 \leq j \leq 2 J, a_{j j}=1-k\left(j+\mu_{j}^{*}\right), a_{j, j-1}=j k$; and for $2 \leq j \leq J, a_{j, 2 j}=4 k b_{2 j}$. The values in the first row, which employs a different formula, are given by $a_{11}=\exp \left(-k \mu_{1}^{*}\right)+8 k^{2} b_{2}, a_{12}=$ $4 k b_{2}\left(1-k\left(2+\mu_{2}^{*}\right)\right)$ and $a_{14}=(4 k)^{2} b_{2} b_{4}$. Of course, $\mathbf{U}^{0}$ is the vector with the initial approximation to $\varphi$ in (1.2).

Thus, we define again the local discretization error, $\boldsymbol{\tau}^{n+1}=\left(\tau_{1}^{n+1}, \ldots, \tau_{2 J}^{n+1}\right)$, $0 \leq n \leq N-1$ as

$$
\boldsymbol{\tau}^{n+1}=\frac{1}{k}\left(\mathbf{u}^{n+1}-\mathcal{A}(k, h) \mathbf{u}^{n}\right), 0 \leq n \leq N-1,
$$

and the global discretization error as $\mathbf{E}^{n}=\left(E_{1}^{n}, \ldots, E_{2 J}^{n}\right), E_{j}^{n}=u_{j}^{n}-U_{j}^{n}$, $1 \leq j \leq 2 J, 0 \leq n \leq N$. Then,

$$
\mathbf{E}^{n+1}=\mathcal{A}(k, h) \mathbf{E}^{n}+k \boldsymbol{\tau}^{n+1}, \quad 0 \leq n \leq N-1 .
$$

And, we carry out the consistency analysis and the boundedness of $\|\mathcal{A}(k, h)\|_{\infty}$ to obtain the convergence. For a vector $\mathbf{v}=\left(v_{1}, \ldots, v_{2 J}\right)$, we denote by $\|\mathbf{v}\|_{\infty}$ its maximum norm. From now on, $C$ will denote a positive constant which is independent of $k, n(0 \leq n \leq N)$ and $h, j(1 \leq j \leq 2 J)$; $C$ possibly has different values in different places. 
Lemma 4 (Stability). Let $\mu^{*}$ have bounded and continuous first derivatives on $(0,1], b$ bounded on $(0,1]$, and $r$ a fixed constant, $0<r \leq 1$, with $k=r h$. Then

$$
\|\mathcal{A}(k, h)\|_{\infty} \leq 1+C k .
$$

Proof: $\|\mathcal{A}\|_{\infty}$ is defined as

$$
\|\mathcal{A}(k, h)\|_{\infty}=\max _{1 \leq i \leq 2 J}\left\{\sum_{j=1}^{2 J}\left|a_{i j}\right|\right\} .
$$

Therefore,

$$
\begin{aligned}
\|\mathcal{A}(k, h)\|_{\infty} \leq & \max \left\{e^{-k \mu_{1}^{*}}+8 k^{2} b_{2}+4 k b_{2}\left(1-k\left(2+\mu_{2}^{*}\right)\right)+(4 k)^{2} b_{2} b_{4},\right. \\
& \max _{2 \leq i \leq J}\left\{\left|1-k\left(i+\mu_{i}^{*}\right)\right|+k\left(i+4 b_{2 i}\right)\right\}, \\
& \left.\left.\max _{J+1 \leq i \leq 2 J}\left\{\mid 1-k\left(i+\mu_{i}^{*}\right)\right) \mid+k i\right\}\right\} .
\end{aligned}
$$

On the one hand, we have that function $\mu^{*}$ is continuously differentiable and $b$ is bounded, so

$$
e^{-k \mu_{1}^{*}}+8 k^{2} b_{2}+4 k b_{2}\left(1-k\left(2+\mu_{2}^{*}\right)\right)+(4 k)^{2} b_{2} b_{4} \leq 1+C k .
$$

On the other hand, the boundedness of $\mu^{*}$ and $b$ and the property $r \leq 1$ allows us to write

$$
\begin{aligned}
\left.\mid 1-k\left(i+\mu_{i}^{*}\right)\right) \mid+k\left(i+4 b_{2 i}\right) & \left.=\mid(1-k i)-k \mu_{i}^{*}\right) \mid+k\left(i+4 b_{2 i}\right) \\
& \leq 1+k\left|\mu^{*}\left(x_{i}\right)\right|+4 k b_{2 i} \\
& \leq 1+C k .
\end{aligned}
$$

And, by means of the same argument,

$$
\left|1-k\left(i+\mu_{i}^{*}\right)\right|+k i \leq 1+C k,
$$

which concludes with the estimate (3.10).

Lemma 5 (Consistency). Let functions $\mu^{*}$ and $b$ be $\mathcal{C}^{1}((0,1]), k=r h$, and let $u$ have Lipschitz continuous first derivatives in $(0,1] \times[0, T]$. Then, as $k \rightarrow 0$, the following estimates hold

$$
\left\|\boldsymbol{\tau}^{n+1}\right\|_{\infty}=O(k), \quad 0 \leq n \leq N-1 .
$$


Proof: From (2.6)-(2.7) we can write

$$
\begin{aligned}
\tau_{1}^{n+1} & =\frac{1}{k}\left(u_{1}^{n+1}-\left(\exp \left(-k \mu_{1}^{*}\right) u_{1}^{n}+4 k b_{2} u_{2}^{n+1}\right)\right), \\
\tau_{j}^{n+1} & =\frac{u_{j}^{n+1}-u_{j}^{n}}{k}+X_{j} \frac{u_{j}^{n}-u_{j-1}^{n}}{h}-\left(\mu_{j}^{*} u_{j}^{n}+4 b_{2 j} u_{2 j}^{n}\right), \quad j=2, \ldots, 2 J,
\end{aligned}
$$

Thus, from equation (3.13) we arrive at

$$
\begin{aligned}
\tau_{j}^{n+1}= & \left(u_{t}\right)_{j}^{n}+X_{j}\left(u_{x}\right)_{j}^{n}+\mu_{j}^{*} u_{j}^{n}-4 b_{2 j} u_{2 j}^{n} \\
& +\int_{0}^{1}\left(u_{t}\left(X_{j}, t^{n}+\theta k\right)-u_{t}\left(X_{j}, t^{n}\right)\right) d \theta \\
& +X_{j} \int_{0}^{1}\left(u_{x}\left(X_{j}+\sigma h, t^{n}\right)-u_{x}\left(X_{j}, t^{n}\right)\right) d \sigma, \quad j=2, \ldots, 2 J .
\end{aligned}
$$

and, due to the smoothness properties of $u$, we have

$$
\left|\tau_{j}^{n+1}\right| \leq C(k+h), \quad j=2, \ldots, 2 J .
$$

Finally, from equations (2.3) and (3.12), we have

$$
\begin{aligned}
\tau_{1}^{n+1} & =\frac{1}{k}\left(u\left(X_{1} e^{-k}, t^{n}\right) \exp \left\{-\int_{t^{n}}^{t^{n+1}} \mu^{*}\left(x\left(\tau ; t^{n+1}, X_{1}\right)\right) d \tau\right\}-\exp \left(-k \mu_{1}^{*}\right) u_{1}^{n}\right. \\
& +4 \int_{t^{n}}^{t^{n+1}} \exp \left\{-\int_{\tau}^{t^{n+1}} \mu^{*}\left(x\left(s ; t^{n+1}, X_{1}\right)\right) d s\right\} b\left(2 x\left(\tau ; t^{n+1}, X_{1}\right)\right) u\left(2 x\left(\tau ; t^{n+1}, X_{1}\right), \tau\right) d \tau \\
& \left.-4 k b_{2} u_{2}^{n+1}\right) .
\end{aligned}
$$

Next, we use the regularity properties of $\mu^{*}, b$ and $u$, the convergence properties of rectangular quadrature rules and the mean value theorem to obtain

$$
\begin{aligned}
\left|\tau_{1}^{n+1}\right| & \leq \frac{1}{k}\left(u\left(X_{1} e^{-k}, t^{n}\right)\left|\exp \left\{-\int_{t^{n}}^{t^{n+1}} \mu^{*}\left(x\left(\tau ; t^{n+1}, X_{1}\right)\right) d \tau\right\}-\exp \left(-k \mu_{1}^{*}\right)\right|\right. \\
& \left.+\exp \left(-k \mu_{1}^{*}\right)\left|u\left(X_{1} e^{-k}, t^{n}\right)-u_{1}^{n}\right|\right) \\
& +\frac{4}{k}\left(\int_{t^{n}}^{t^{n+1}} \exp \left\{-\int_{\tau}^{t^{n+1}} \mu^{*}\left(x\left(s ; t^{n+1}, X_{1}\right)\right) d s\right\} b\left(2 x\left(\tau ; t^{n+1}, X_{1}\right)\right) u\left(2 x\left(\tau ; t^{n+1}, X_{1}\right), \tau\right) d \tau\right. \\
& \left.-k b_{2} u_{2}^{n+1}\right) . \\
\leq & C k .
\end{aligned}
$$

Then (3.14)-(3.15) produce the estimate (3.11).

In the following result, we prove the convergence of the numerical method. 
Theorem 6 (Convergence). Under the hypotheses of Lemma 5, if $\left\|\mathbf{E}^{0}\right\|_{\infty}=$ $O(k)$, as $k \rightarrow 0$, then

$$
\left\|\mathbf{E}^{n}\right\|_{\infty}=O(k), \quad 0 \leq n \leq N,
$$

as $k \rightarrow 0$.

Proof: From the definition of the global discretization error, we have

$\mathbf{E}^{n}=\mathcal{A}(k, h) \mathbf{E}^{n-1}+k \boldsymbol{\tau}^{n}=\mathcal{A}(k, h)^{n} \mathbf{E}^{0}+k \sum_{l=0}^{n-1} \mathcal{A}(k, h)^{l} \boldsymbol{\tau}^{n-l}, 1 \leq n \leq N$.

Thus

$\left\|\mathbf{E}^{n}\right\|_{\infty} \leq\left(\|\mathcal{A}(k, h)\|_{\infty}\right)^{n}\left\|\mathbf{E}^{0}\right\|_{\infty}+k \sum_{l=0}^{n-1}\left(\|\mathcal{A}(k, h)\|_{\infty}\right)^{l}\left\|\boldsymbol{\tau}^{n-l}\right\|_{\infty}, 1 \leq n \leq N$.

Therefore, the use of (3.10), (3.11) and $k=r h$ produces (3.16).

\section{Numerical experiments}

We have checked experimentally both numerical methods. The following experiment shows their optimal rate of convergence and allows us to compare their efficiency.

In this first experiment, we suppose that $\mu(x)=\nu(x)$. This situation also includes the case in which there is neither cellular death $(\mu(x)=0)$ nor migration $(\nu(x)=0)$. Now, following the work in the case of a positive minimal division size [4], we take the size-specific division rate function as

$$
b(x)=2^{6} x^{3}(1-x)^{3}, \quad 0<x \leq 1,
$$

and the initial data employed in [3] with $m=4$

$$
\varphi(x)=C(1-x) x^{3} \sin (\pi(4 x+1)+1), \quad 0<x \leq 1,
$$

where $C$ is chosen to obtain $\max _{0 \leq x \leq 1}(\varphi(x))=1$.

We have carried out an extensive simulation with both numerical schemes (UW and NGM) for different values of the discretization parameters. In this experiment we compute the solution until the final time $T=1$. In this test problem, we do not know the analytical solution so, in order to compare with the numerical solution, we take as the exact solution the computed approximation with a sufficiently small value of the size step $k$ (and $h$ where it is necessary). We have employed $k^{*}=2.441406 e-4$ in the case of the 
natural grid method (NGM), and $h^{*}=k^{*}=3.05176 e-5$ for the upwind method (UM).

With respect to the natural grid method, for each $k$, we compare at every time step $t^{n}=n k, 0 \leq n \leq N$, the numerical solution computed by the NGM, $\mathbf{U}_{k}^{n}, 0 \leq n \leq N$, with the representation of the solution corresponding to $k^{*}$ at the coarsest grid obtained with $k$, and the corresponding time levels $t^{n}: \tilde{\mathbf{U}}_{k}^{n}$. This is made with the global error computed as,

$$
e_{k}=\max _{0 \leq n \leq N}\left\|\mathbf{U}_{k}^{n}-\tilde{\mathbf{U}}_{k}^{n}\right\|_{\infty} .
$$

Left-hand side plot in Figure 1 shows the error obtained with the values of $k=6.25 e-2, k=3.125 e-2, k=1.5625 e-2, k=7.8125 e-3$, $k=3.90625 e-3, k=1.95313 e-3$ and $k=9.76563 e-4$ (dashed line). Experimentally, we observe that the NGM is a first-order method, verifying the theoretical analysis made in Theorem 3.
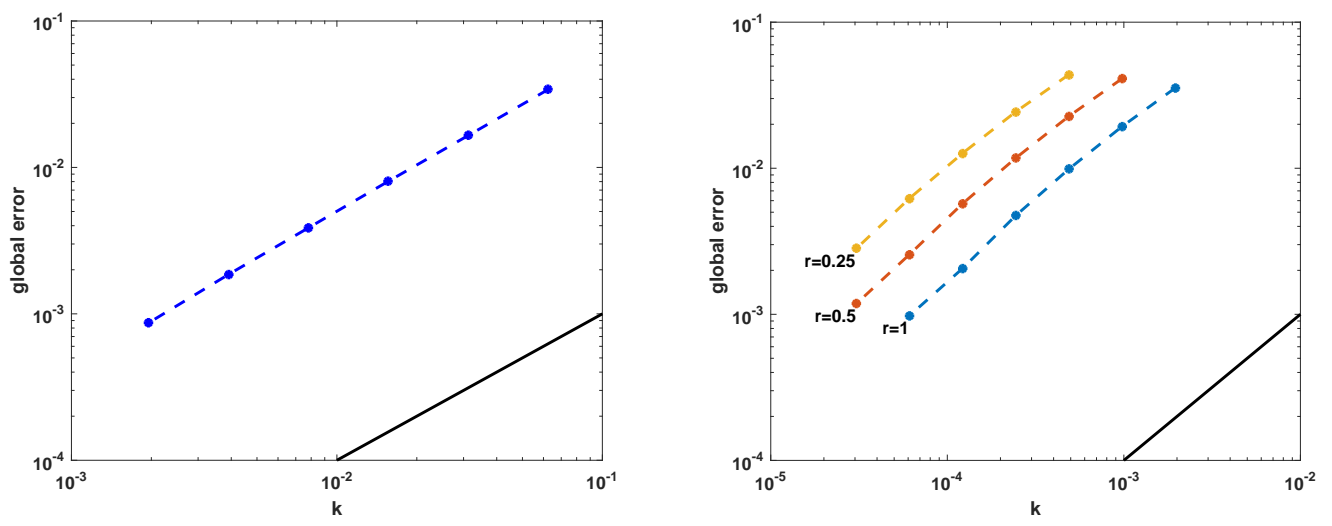

Figure 1: Global error plots.

On the left: Errors obtained with the NGM (dashed line). Solid line shows order 1.

On the right: Errors obtained with the UM with different values of $r$ (dashed lines). Solid line shows order 1 .

In the case of the upwind method, for each $h$ and $k$, we compare at every time step $t^{n}=n k, 0 \leq n \leq N$, the numerical solution computed by the UM, $\mathbf{U}_{k, h}^{n}, 0 \leq n \leq N$, with the representation of the solution corresponding to $h^{*}$ and $k^{*}$ at the coarsest grid obtained with $h$ and $k$, and the corresponding time levels $t^{n}: \tilde{\mathbf{U}}_{k, h}^{n}$. This is made with the global error computed as,

$$
e_{k, h}=\max _{0 \leq n \leq N}\left\|\mathbf{U}_{k, h}^{n}-\tilde{\mathbf{U}}_{k, h}^{n}\right\|_{\infty}
$$

Right-hand side plot in Figure 1 shows the results obtained with different values of $r$ and different values of $k(r=1: k=1.95313 e-3, k=9.76563 e-$ 
$4, k=4.88281 e-4, k=2.44141 e-4, k=1.22070 e-4$ and $k=6.10352 e-5$; $r=0.5: k=9.76563 e-4, k=4.88281 e-4, k=2.44141 e-4, k=1.22070 e-$ $4, k=6.10352 e-5$ and $k=3.05176 e-5$; and $r=0.25: k=9.76563 e-4$, $k=2.44141 e-4, k=1.22070 e-4, k=6.10352 e-5$, and $k=3.05176 e-5)$. Note that the method is of first-order, experimentally, corroborating the theoretical analysis demonstrated in Theorem 6 . Besides, we have observed that the method is not able to obtain the solution of the problem when the CFL condition is not satisfied. This situation is shown in Lemma 4, where the condition $r \leq 1$ is needed for the convergence of the method.

In order to compare the efficiency of the numerical methods, it is usual to analyze it through log-log efficiency charts: the vertical axis corresponds to the error and the horizontal axis is the computational cost. So, for different values of the discretization parameters, we plot the error produced by the corresponding approximation versus the computational effort required. In Figure 2, we show efficiency plots for the numerical methods. In the plot on the left, we compare the UM results for different values of $r$. We observe that the most efficient relationship among $k$ and $h$ is $r=1$. Then, in the plot on the right, we compare the results obtained with the NGM and the values collected with such a best relationship of the spatial and time discretization parameters $(r=1)$ in the UM. It is clear that this best relationship in the UM is more efficient than the NGM.
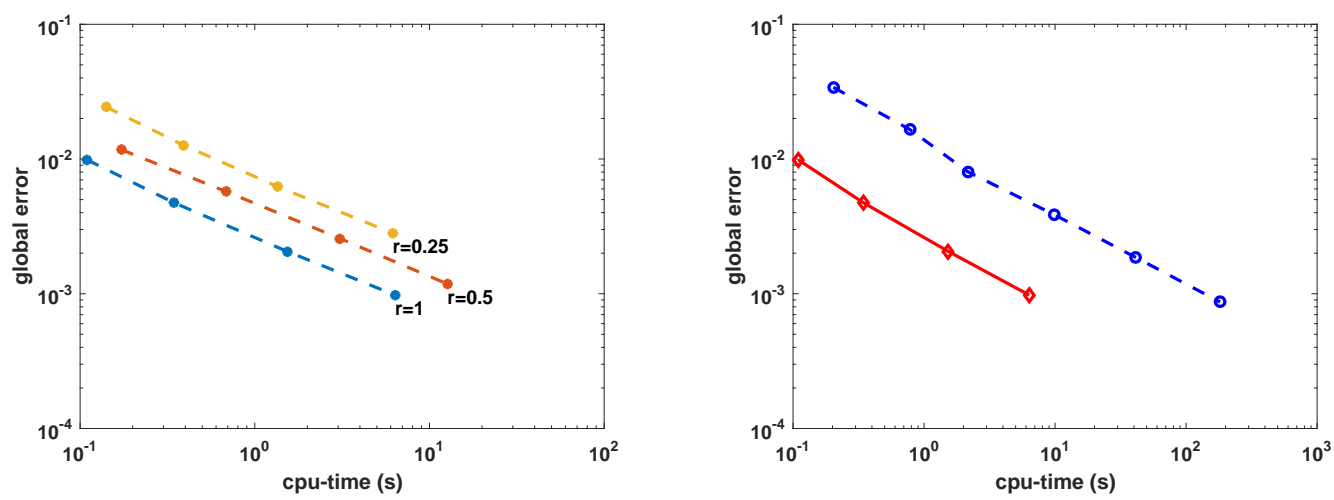

Figure 2: Efficiency plots.

On the left: UM with different values of $r$.

On the right: UM solid line $(r=1)$, NGM dashed line.

We also consider a second experiment in which we analyze the dynamics of the solution depending on the mortality and migration rates, therefore we consider $\mu(x)=\nu(x)+C, C \in \mathbb{R}$, in order to see the different behavior in the population. We do not modify the other functions in the previous 
experiment. The hypotheses that allow the population to arrive to a stable situation (extinction) were introduced in [14]. However, other situations were not studied theoretically. Here, we have carried out an extensive simulation with both numerical schemes for a long time integration. Numerically, we have observed only two possible behaviors depending on $C$ : extinction or an unbounded population. More precisely, for different values of the parameter $C \in[-2,4]$, we have computed $t_{u p}$ : the time at which the approximated total population is higher than $1 e+20$ (unbounded solution), or $t_{\text {down }}$ : the time at which it is lower than $1 e-20$ (extinction). Figure 3 shows these times $\left(t_{\text {up }}\right.$ or $\left.t_{\text {down }}\right)$ obtained by means of the UM with $k=6.1035 e-05$ and $r=1$ (NGM provides similar values). We observe a change in the dynamics of the solution for an estimated value of the parameter $C^{*} \approx 0.261$. As a conclusion, the balance in the population ruled by the partial differential equation (1.1) allows us to assure extinction for $C>C^{*}$ and an unbounded population for $C<C^{*}$.

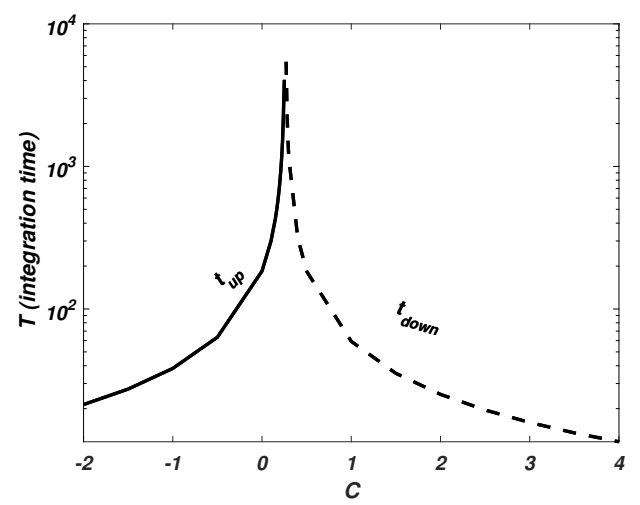

Figure 3: Behavior of the solution depending on $C . t_{u p}$ (solid line): time at which the approximated total population is higher than $1 e+20$ (unbounded solution). $t_{\text {down }}$ (dashed line): time at which it is lower than $1 e-20$ (extinction). Time is represented in logarithm scale.

Finally, we declare that all the calculations were carried out using double precision arithmetic on a personal computer with i7-4790 CPU.

\section{Conclusions}

The study of cell populations by means of the use of structured population models, and their numerical simulation, are current and important topics.

In this work, we deal with a specific problem that describes the dynamics of a size-structured cell population, in which the reproduction process is 
considered to be by division into two equal parts. A remarkable point is that the division of all-sizes cells is allowed. This characteristic enables to consider the phenomena of "dwarfism" and its application to the description of the dynamics of thalassemia disease.

Its mathematical description is based on an initial value problem for a first order partial differential equation without boundary conditions, because the recruitment is made by either fission or other mechanisms that could occur within an open system (controlled by $\nu(x)$ ). We have designed two numerical methods to attain the solution to such model. They are based on proposals that we developed previously in the case of the existence of a positive minimal size of division. To our best knowledge, they are the only numerical methods considered in the literature for this model. Both of them are specially adapted to the features of the model and prevent the use of the values at the characteristic $x=0$, in contrast with other general numerical techniques as, for instance, general finite difference and finite element methods.

The proposed schemes are based on different procedures. On one hand, the integration along the characteristic curves of the problem with the use of the natural grid. On the other hand, a finite different method (with an upwind technique). Both are completely analyzed: we have proved their first-order convergence using their consistency and stability properties. Also, we carried out numerical experimentation that corroborates our theoretical results. The comparison of both methods shows that, for this problem, the first order UM with $r=1$ offers much better approximations than the NGM at the same computational cost. Finally, these methods allow to describe the dynamics of the solution.

\section{Acknowledgments}

Authors are very grateful to the referees for their careful reading of the original manuscript and their aid to improve it.

This work was supported in part by projects MTM2014-56022-C2-2-P and MTM2017-85476-C2-1-P of the Spanish Ministerio de Economía y Competitividad and European FEDER Funds. Also by project VA138G18 of the Consejería de Educación, Junta de Castilla y León, Spain; and project VA041P17 of the Consejería de Educación, Junta de Castilla y León and European FEDER Funds.

\section{References}

[1] L. Abia, O. Angulo, J. C. López-Marcos, Size-structured population dynamics models and their numerical solutions, Discrete Contin. Dyn. Syst. Ser. B 4 (2004) 1203-1222. 
[2] L.M. Abia, O. Angulo, J. C. López-Marcos, M. A. López-Marcos, Numerical schemes for a size-structured cell population model with equal fission, Math. Comp. Model. 50 (2009) 653-664.

[3] L. M. Abia, O. Angulo, J. C. López-Marcos, M. A. López-Marcos. A numerical study on the estimation of the stable size distribution for a cell population balance model, Math. Meth. Appl. Sci. 41 (2018) 28942905 .

[4] O. Angulo, J. C. López-Marcos, M. A. López-Marcos, A second-order method for the numerical integration of a size-structured cell population model, Abstr. Appl. Anal. 549168 (2015) 1-8.

[5] O. Angulo, J. C. López-Marcos, M. A. López-Marcos, A second-order numerical method for a cell population model with asymmetric division, J. Comp. Appl. Math. 309 (2017) 522-531.

[6] G. I. Bell, E. C. Anderson, Cell growth and division: I. a mathematical model with applications to cell volume distributions in mammalian suspension cultures, Biophys. J. 7 (1967) 329-351.

[7] O. Diekmann, H. J. A. M. Heijmans, H. R. Thieme, On the stability of the cell size distribution, J. Math. Biol. 19 (1984) 227-248.

[8] S. El Mourchid, G. Metafune, A. Rhandi, J. Voigt, On the chaotic behaviour of size structured cell populations, J. Math. Anal. Appl. 339 (2008) 918-924.

[9] R. Galanello, A. Cao, $\alpha$-thalassemia, Genetics in Medicine 13 (2011) 83-88.

[10] G. Geiner, R. Nagel, Growth of cell populations via one parameter semigroups of positive operators, in: J. Goldstein, S. Rosencrans, G. Sod (Eds), Mathematical applied to science, Academic Press, London, 1988, pp. $79-104$.

[11] P. J. Giardina, S. Rivella, Thalassemia syndromes, In R. Hoffman, E. J. Benz, L. E. Silberstein, H. E. Heslop, J. I. Weitz, J. Anastasi (Eds.), Hematoloygy: Basis Principles and Practice, 6th ed, Elsevier, Philadelphia, PA, 2013, Chapter 38.

[12] H. J. A. M. Heijmans, On the Stable Size Distribution of Populations Reproducing by Fission into Two Unequal Parts, Math. Biosci. 12 (1984) 119-150. 
[13] M. Iannelli and F. Milner. The Basic Approach to Age-Structured Population Dynamics. Springer, The Netherlands, 2017.

[14] K. H. Howard, A size-structured model of cell dwarfism, Disc. Cont. Dyn. Sys. B 1 (2001) 471-484.

[15] K. Ito, F. Kappel, G. Peichl, A fully discretized approximation scheme for size-structured population models, SIAM J. Numer. Anal. 28 (1991) 923.

[16] D. Ramkrishna, Population balances: theory and applications to particulate systems in engineering, Academic Press, San Diego, 2000.

[17] A.D. Randolph, A population balance for countable entities, Can. J. Chem. Eng. 42 (1964) 280-281.

[18] R. Rudnicki, Chaoticity and invariant measures for a cell population model, J. Math. Anal. Appl. 393 (2012) 151-165.

[19] D.P. Steensma, R.J. Gibbons, D.R. Higgs, Acquired $\alpha$-thalassemia in association with myelodysplastic syndrome and other hematologic malignancies, Blood 105 (2005) 443-452. 